Meta

Journal des traducteurs

Translators' Journal

\title{
La traduction et l'interprétatin dans les Territoires du Nord-Ouest
}

\section{Madeleine Daigle}

Volume 38, numéro 1, mars 1993

La traduction et l'interprétation dans le nord du Canada

Translation and Interpretation in Northen Canada

URI : https://id.erudit.org/iderudit/002444ar

DOI : https://doi.org/10.7202/002444ar

Aller au sommaire du numéro

Éditeur(s)

Les Presses de l'Université de Montréal

ISSN

0026-0452 (imprimé)

1492-1421 (numérique)

Découvrir la revue

Citer cet article

Daigle, M. (1993). La traduction et l'interprétatin dans les Territoires du Nord-Ouest. Meta, 38(1), 107-110. https://doi.org/10.7202/002444ar
Résumé de l'article

Depuis que le CTIC a vu le jour, en 1975, les interprètes et les traducteurs du Canada travaillant dans les deux langues officielles peuvent en devenir membres. Bien que l'interprétation et la traduction dans les langues autochtones aient été développées depuis de nombreuses années, le Bureau des langues du gouvernement des Territoires du Nord-Ouest fut seulement fondé dans les années soixante-dix. La dernière décennie a vu une nette amélioration des services d'interprétation et de traduction dans le nord du Canada, comme en témoigne la création de l'ITSNWT (Société d'interprétation et de traduction des Territoires du Nord-Ouest). La société met actuellement au point des examens officiels afin que les interprètes et traducteurs puissent être diplômés en langues autochtones.
Ce document est protégé par la loi sur le droit d'auteur. L’utilisation des services d’Érudit (y compris la reproduction) est assujettie à sa politique d'utilisation que vous pouvez consulter en ligne.

https://apropos.erudit.org/fr/usagers/politique-dutilisation/ 


\title{
LA TRADUCTION ET L'INTERPRÉTATION DANS LES TERRITOIRES DU NORD-OUEST
}

\author{
MADELEINE DAIGLE \\ Culture et Communications, Yellowknife, \\ Territoires du Nord-Ouest, Canada
}

\begin{abstract}
Rêsumé
Depuis que le CTIC a vu le jour, en 1975, les interprètes et les traducteurs du Canada travaillant dans les deux langues officielles peuvent en devenir membres. Bien que l'interprétation et la traduction dans les langues autochtones aient été développées depuis de nombreuses années, le Bureau des langues du gouvernement des Territoires du Nord-Ouest fut seulement fondé dans les années soixante-dix. La dernière décennie a vu une nette amélioration des services d'interprétation et de traduction dans le nord du Canada, comme en témoigne la création de l'ITSNWT (Société d'interprétation et de traduction des Territoires du Nord-Ouest). La société met actuellement au point des examens officiels afin que les interprètes et traducteurs puissent être diplômés en langues autochtones.
\end{abstract}

\begin{abstract}
Since the birth of CTIC in 1975, interpreters and translators in Canada's two official languages have had the benefit of joining this association. Although interpretation and translation in aboriginal languages has been going on for many years, it was not until the 1970's that the Language Bureau was set up in the Northwest Territories. Over the last decade much improvement in services has taken place in the field of interpreting and translation in Canada's North, including the creation of ITSNWT, the interpreter/translation Society of the Northwest Territories formed in 1989. Currently the Society is in the process of setting up formal examinations to certify interpreters and translators in the native language.
\end{abstract}

Il n'est nul besoin d'écrire des pages entières pour comprendre pourquoi le Canada est une terre fertile pour toutes les professions langagières, surtout en ce qui concerne la traduction et l'interprétation. Partout au Canada, des associations professionnelles regroupant des traducteurs, des interprètes et des terminologues ont été mises sur pied. Ces différentes associations provinciales visent principalement à protéger les intérêts de leurs membres et du public - utilisateur direct ou indirect de textes traduits. II n'est donc pas étonnant que ces associations aient senti le besoin de se doter d'un organisme central pour représenter la profession à l'échelle nationale. Le Conseil des traducteurs et des interprètes du Canada (CTIC) a donc vu le jour afin, entre autres raisons, d'assurer l'uniformité des normes dans l'exercice de la profession. Depuis 1975, le CTIC administre, en collaboration avec les associations membres, l'examen d'agrément en traduction dans les deux langues officielles du pays.

Aujourd'hui, le Canada ouvre ses portes à un nombre de plus en plus élevé d'immigrants appartenant à un groupe linguistique autre que l'anglais ou le français, d'où un accroissement de la demande de services de traduction et d'interprétation en langues dites «étrangères». Il ne faut donc pas être surpris de voir des interprètes et des traducteurs, travaillant avec des combinaisons linguistiques autres que l'anglais et le français, se joindre à une association de professionnels en vue d'obtenir le même statut que leurs collègues spécialistes des langues officielles du pays. 
Alors qu'à l'échelle nationale on se concentre principalement sur les langues officielles et celles dites «étrangères», il se fait depuis longtemps déjà de la traduction et de l'interprétation dans des langues souvent méconnues qui, loin d'être «étrangères», appartiennent aux peuples habitant depuis très longtemps un «coin de pays» couvrant plus du tiers de la superficie totale du Canada. Nous faisons référence ici aux Territoires du Nord-Ouest.

Avant de poursuivre, il est utile de brosser un tableau sociolinguistique de ces territoires et d'en donner un bref aperçu historique. Les deux principaux groupes autochtones des Territoirs du Nord-Ouest sont les Inuit, concentrés en majeure partie dans l'est de l'Arctique, et les Dénés, vivant dans la partie ouest. Environ $70 \%$ de la population totale des Territoires du Nord-Ouest appartient à l'un ou à l'autre de ces groupes. La principale langue des Inuit, l'inuktitut, se parle dans l'est de l'Arctique et le long de la côte arctique, alors que, dans l'ouest de l'Arctique, on utilise surtout l'inuvialuktun et l'innuinaqiun. Les cinq principales langues dénés, parlées surtout dans la vallée du fleuve Mackenzie, sont le flanc-de-chien ou Dogrib, la langue saulteuse ou le Chipewyan, le loucheux ou le Gwich'in, l'esclave du nord ou le North Slavey et l'esclave du sud ou le South Slavey. Environ une cinquantaine de personnes vivant dans la région de Fort Smith, située au sud du Grand Lac des Esclaves, parlent le cri.

Jusqu'en 1946, le Conseil des Territoires du Nord-Ouest, composé de hauts fonctionnaires du gouvernement fédéral, dirigeait et administrait ces territoires; il n'est donc pas surprenant qu'on y ait adopté l'anglais comme langue administrative et gouvernementale. Petit à petit, des députés élus remplacèrent les hauts fonctionnaires nommés par Ottawa et, dès 1966, les membres élus constituaient la majorité du Conseil, avec sept membres élus par la population des Territoires et cinq nommés par Ottawa. À la même époque, les premiers membres élus de l'est de l'Arctique, y compris le premier Inuk («Inuk» est le singulier d'«Inuit»), prirent place au Conseil. Au début de 1967, le siège du gouvernement passa d'Ottawa à Yellowknife et on créa une fonction publique territoriale. Le premier Conseil entièrement élu entra en fonction en 1975. Les députés dénés, métis et inuit détenaient la majorité des 15 sièges du Conseil qui devint, après 1976, l'Assemblée législative. Aujourd'hui, l'Assemblée compte 24 députés, dont la majorité est d'ascendance autochtone. Ces députés peuvent recourir à des services d'interprétation en quatre langues dénés, en deux langues inuit et en français.

Quant à la langue d'enseignement, ce fut longtemps le français, entre autres dans la partie sud-ouest des Territoires, pour la simple raison que les missionnaires oblats, Français pour la plupart, s'occupèrent de l'éducation des autochtones dénés. Chez les Inuit, les missionnaires anglicans se chargèrent de l'enseignement qui se faisait en anglais et en inuktitut. Ce n'est qu'à la fin des années cinquante que le gouvernement des Territoires du Nord-Ouest créa son propre ministère de l'Éducation et assuma la responsabilité de l'enseignement pour les Territoires. L'anglais devint la langue d'enseignement, mais, parallèlement, on continua l'enseignement de l'inuktitut dans l'est de l'Arctique.

Nous nous sommes donc retrouvés devant une population utilisant principalement l'anglais. Dans un tel contexte, il n'est pas étonnant qu'il se soit toujours fait de l'interprétation dans les langues autochtones, que ce soit de l'interprétation «maison», les enfants scolarisés en anglais interprétant pour des parents autochtones unilingues, ou, de façon plus officielle, avec la mise sur pied du Bureau des langues au sein du ministère de la Culture et des Communications du gouvernement des Territoires du Nord-Ouest, au début des années soixante-dix. À cette époque, trois interprètes-traducteurs inuit offraient en permanence des services de traduction et d'interprétation à l'Assemblée législative, qui comptait alors neuf députés autochtones élus, et quatre interprètes du groupe des langues dénés travaillaient sur une base temporaire. On offrait également des services de traduction et d'interprétation devant les tribunaux et au sein d'un bon nombre de ministères. 
Ces interprètes et ces traducteurs apprenaient leur «métier» sur le tas et ceux qui travaillaient pour le gouvernement des Territoires du Nord-Ouest recevaient une formation plus structurée du Bureau des langues.

En 1984, le gouvernement des Territoires du Nord-Ouest introduisit la Loi sur les langues officielles, faisant du français et de l'anglais les langues officielles des Territoires, tout en reconnaissant l'existence de sept langues autochtones. La demande pour des services de traduction et d'interprétation en langues autochtones augmenta considérablement, de même que le nombre de traducteurs et d'interprètes. On en compte aujourd'hui plus de soixante-dix dans les Territoires. Le Bureau des langues continue d'offrir une formation aux traducteurs et interprètes à son emploi. Parallèlement à ce dernier, le Collège de l'Arctique situé à Fort Smith et à Iqaluit a mis sur pied un programme d'études en traduction et en interprétation des langues dénés et inuit. Ce programme, d'une durée d'un an (on prévoit un prolongement d'une autre année), existe depuis bientôt trois ans.

Au niveau des tribunaux, bien que les procès se déroulent en anglais, les avocats, les juges et les greffiers se retrouvent souvent devant des accusé(e)s ou des témoins unilingues autochtones. On doit donc faire appel à des interprètes afin d'assurer la communication entre témoins, accusé(e)s, membres du jury, avocats et juges. Les Territoires du Nord-Ouest se sont vite retrouvés devant un problème: où trouver des interprètes en langues autochtones expérimentés dans le domaine juridique? Lorsque le procès se déroule dans une communauté où il y a une division régionale du Bureau des langues du gouvernement des Territoires du Nord-Ouest, la Cour fait appel à ce service pour trouver des interprètes chevronnés. Par contre, il est beaucoup plus difficile d'en trouver dans les plus petites communautés. Le problème s'aggrava en 1986 quand le ministre de la justice déposa un projet de loi modifiant la Loi sur le jury, afin de permettre à des autochtones unilingues d'être membre d'un jury. On s'est donc retrouvé avec des jurés unilingues devant statuer sur une cause se déroulant principalement en anglais et selon les règles d'un système juridique différent de celui des autochtones. On ne tarda pas à sentir le besoin de former des interprètes judiciaires afin de surmonter ce problème. Il y a environ trois ans, le ministère de la Justice a mis sur pied un programme de formation à l'intention des interprètes judiciaires autochtones. Ce programme porte fruit, car on commence déjà à sentir une amélioration au niveau de la qualité de l'interprétation.

En octobre 1990, le gouvernement modifia la Loi sur les langues officielles de manière à accorder le statut de langues officielles à six langues autochtones. A la suite de cette modification, les Territoires du Nord-Ouest devenaient officiellement «octolingues». Comme l'histoire du Canada a su le prouver, la traduction et l'interprétation en langues autochtones continueront à se developper et on verra un plus grand nombre de traducteurs et d'interprètes travaillant dans ces langues.

Bien avant que les langues autochtones ne reçoivent le statut de langues officielles, et en prévision d'une demande accrue de services dans ces langues, on s'était déjà rendu compte du besoin de créer un système permettant de reconnaître les compétences des traducteurs et des interprètes en langues autochtones. Le Bureau des langues octroie un certificat à ses employé(e)s qui reçoivent une formation au travail et le collège de l'Arctique en décerne un après une année de formation. Mais il n'existe aucun organisme, que ce soit dans les Territoires du Nord-Ouest ou ailleurs au Canada, qui soit en mesure d'agréer les professionnels de la traduction et de l'interprétation en langues autochtones.

En mai 1989, suite aux recherches intensives de linguistes et de spécialistes des langues autochtones sur la façon de mettre en place des mécanismes permettant de reconnaître officiellement les compétences professionnelles des traducteurs et des interprètes en langues autochtones, un groupe de traducteurs et d'interprètes s'est réuni pour étudier la formation d'une association professionnelle, dont le principal mandat serait d'assurer 
l'uniformité des normes dans l'exercice de leur métier et d'obtenir ainsi le même statut que leurs pairs du «Sud». La différence existant entre l'interprétation et la traduction dans les Territoires et dans le reste du Canada porte bien davantage sur le choix des combinaisons linguistiques que sur les gens exerçant le métier. Aussi a-t-on décidé de former une association qui deviendrait éventuellement membre d'un organisme reconnu à l'échelle nationale, dont la principale activité consiste à agréer des traducteurs et des interprètes au Canada. L'Interpreters / Translators Society of the Northwest Territories (ITSNWT) a vu le jour au cours de cette réunion et s'est tout de suite dotée d'un conseil d'admisnistration composé de huit personnes, chacune représentant une des langues en usage au Bureau des langues. Il fallut rédiger le règlement interne, enregistrer la société et présenter une demande officielle d'adhésion au Conseil des traducteurs et des interprètes du Canada. À la suite de cette demande, le CTIC invita la ITSNWT à assister à sa réunion semi-annuelle d'automne. Sans se faire prier, la ITSNWT mandata la présidente de l'association pour se rendre à Ottawa et y faire une présentation officielle. On accepta d'emblée la nouvelle société, non sans d'abord modifier le règlement interne du Conseil afin d'y ajouter «association territoriale» (le CTIC ne comptait, au nombre de ses associations membres, que des associations provinciales). La ITSNWT est la première association territoriale à représenter des traducteurs et des interprètes dont la majorité travaille en langues autochtones. En mars 1990, lors de la réunion du CTIC du printemps, la ITSNWT fut officiellement acceptée en tant qu'association membre. On peut maintenant respirer. Cependant, cette première étape, bien qu'importante, ne signifie pas que le travail est terminé, bien au contraire, elle n'en marque que le début.

Alors que, dans le reste du pays, des mécanismes existent déjà pour agréer les interprètes et les traducteurs en langues officielles et dans les principales langues «étrangères», rien de la sorte n'est en place pour les langues autochtones du pays. La ITSNWT représente les gens qui travaillent dans le domaine: c'est là que le travail doit se faire. La ITSNWT a donc beaucoup de pain sur la planche.

Après plusieurs rencontres, le Conseil d'administration de la ITSNWT a établi ses priorités et, ayant à l'esprit la culture orale plutôt qu'écrite des autochtones, a décidé de mettre sur pied une première série d'examens portant exclusivement sur l'interprétation. Par suite, la société a créé un comité qui avait pour mandat la rédaction de lignes directrices pour les examens d'agrément des traducteurs et des interprètes en langues autochtones. Le Comité d'examens devra encore fixer les conditions d'admissibilité à l'examen d'agrément, déterminer les combinaisons linguistiques des examens (seulement à partir de la liste des membres autochtones, il y a à l'heure actuelle 12 combinaisons linguistiques différentes), choisir et former des évaluateurs pour chaque combinaison linguistique, trouver des textes enregistrés sur bande sonore ou vidéo se prêtant bien à une situation d'interprétation, élaborer les grilles d'évaluation, tenir une séance préliminaire d'examens afin d'évaluer le processus et y apporter les corrections nécessaires. À l'heure actuelle, on prévoit préparer des examens pour l'interprétation simultanée et l'interprétation consécutive d'une langue autochtone vers l'anglais et de l'anglais vers une langue autochtone. Une fois ces tâches accomplies, la ITSNWT sera prête à administrer de manière officielle une première série d'examens en vue d'agréer des interprètes de langues autochtones.

Comme vous pouvez le constater, les Territoires du Nord-Ouest ont plus d'une longueur d'avance en ce qui concerne les langues autochtones. En modifiant la Loi sur les langues officielles, le gouvernement leur a accordé un statut légal qui, en plus de contribuer à la préservation de ces langues, garantira aux résident(e)s autochtones une gamme plus étendue de services dans leurs langues. Grâce aux examens d'agrément de la ITSNWT, on pourra compter sur des services d'interprétation et de traduction de qualité. 